\title{
MINIMUM SIZED FIBRES IN DISTRIBUTIVE LATTICES
}

\author{
DWIGHT DUFFUS and BILL SANDS
}

(Received 20 October 1999; revised 8 November 2000)

Communicated by B. A. Davey

\begin{abstract}
A subset $F$ of an ordered set $X$ is a fibre of $X$ if $F$ intersects every maximal antichain of $X$. We find a lower bound on the function $f(D)$, the minimum fibre size in the distributive lattice $D$, in terms of the size of $D$. In particular, we prove that there is a constant $c$ such that

$$
f(D) \geq c \cdot|D|^{1 / \sqrt{3 \log _{2}|D|}} .
$$

In the process we show that minimum fibre size is a monotone property for a certain class of distributive lattices. This fact depends upon being able to split every maximal antichain of this class of distributive lattices into two parts so that the lattice is the union of the upset of one part and the downset of the other.

2000 Mathematics subject classification: primary 06A07, $06 \mathrm{D} 99$.

Keywords and phrases: distributive lattice, fibre, maximal antichain, splitting property.
\end{abstract}

\section{Introduction}

All ordered sets and lattices in this paper are finite, unless otherwise stated. A subset of an ordered set which meets every maximal chain is called a cutset and one which meets every maximal antichain is called a fibre. It is interesting to investigate the possible sizes of minimal fibres and cutsets in various families of ordered sets. For instance, if we restrict ourselves to $2^{n}$, the Boolean lattice of all subsets of an $n$-element set, and consider cutset sizes, the situation is settled. The minimum size of a cutset is 1 (use either $\emptyset$ or the entire set $[n]$ ) while it is known that minimal cutsets can contain almost all elements of $2^{n}[10]$. Recent papers $[3,4]$ contain work on minimal widths of cutsets in truncated Boolean lattices.

However, in this paper it is fibres which will concern us. Motivated by problems raised in [2], Lonc and Rival [12] made several conjectures concerning sizes of minimal

(C) 2001 Australian Mathematical Society $0263-6115 / 2001 \$ A 2.00+0.00$ 
fibres in $\mathbf{2}^{n}$. In [6], with Winkler, we obtained a number of results for fibres in $\mathbf{2}^{n}$; in particular, we found lower bounds for the minimum and maximum sizes of minimal fibres. As noted in [12], for any ordered set $X$ and any $x \in X$, the set $C(x)$ of all elements of $X$ comparable to $x$ is a fibre and is a minimal one if $X$ is a Boolean lattice and $x \neq 0_{X}$ or $1_{X}$, the minimum and maximum elements of $X$. So, the minimum fibre size for $2^{n}$ is at most $2^{n / 2+1}-1$, for $n$ even, or $3\left(2^{(n-1) / 2}\right)-1$, for $n$ odd, which is the size of $C(x)$ when $x$ is a 'middle-level' element of $2^{n}$. Lonc and Rival conjectured that this is precisely the minimum fibre size. This conjecture is still open.

In the other direction, for any ordered set every fibre must be at least as large as the largest number of pairwise disjoint maximal antichains, so in particular, if the ordered set is graded, fibre size must be at least the length. In general there is no relation between fibre size and the length of maximal chains; for example, the ordered set which is the union of the chains $x_{1}<x_{2}<\cdots<x_{n-1}<x_{n}$ and $y_{1}<y_{2}<\cdots<y_{n}$ and with the further covering relation $x_{n-1}<y_{2}$ has a two-element fibre $\left\{x_{n}, y_{1}\right\}$ although the shortest maximal chain has $n$ elements. For the class $\left\{2^{n}: n \geq 1\right\}$ of Boolean lattices, fibre size is much larger than length, though; in fact, the minimum fibre size is exponential in $n-$ it was shown in [6] that every fibre must have order of magnitude at least $(5 / 4)^{n}$ elements. This has been improved by Łuczak to $(\sqrt[3]{2})^{n}[13]$ using another result from [6]. We include a proof of Łuczak's bound in Section 4.

What about more general lattices? Note that there are (nondistributive) lattices of arbitrary size having a three-element fibre. Just let $L$ be any lattice, and form the lattice $L^{\prime}=L \cup\{a\}$ where $0_{L}<a<1_{L}$ but $a$ is incomparable to all other elements of $L$; then $\left\{0_{L}, a, 1_{L}\right\}$ is a fibre of $L^{\prime}$ regardless of the size of $L$. Nothing interesting here.

However, for the class of all distributive lattices there may be something yet to say. Of course, for some distributive lattices, namely chains, the entire lattice is the only fibre, so we will get no nontrivial upper bound on minimal fibre size. But determining the lower bound for minimum fibre size for distributive lattices remains an interesting problem.

For a distributive lattice $D$, let $f(D)$ denote the minimum fibre size in $D$. As indicated above, we know that $f\left(2^{n}\right)$ is of an order between the cube root and the square root of $2^{n}$. For distributive lattices in general, we can prove the following.

THEOREM 1. There is a constant $c>0$ such that, for any distributive lattice $D$,

$$
f(D) \geq c \cdot|D|^{1 / \sqrt{3 \log _{2}|D|}} .
$$

An easy manipulation of the lower bound shows that

$$
f(D) \geq c \cdot 2^{\sqrt{\left(\log _{2}|D|\right) / 3}}
$$


so $f(D)$ grows to infinity with some alacrity. Still, this is a far cry from what we believe to be true, and what would be the natural extension of the situation for Boolean lattices:

CONJECTURE 1. There exists a constant $\beta>0$ such that $f(D) \geq|D|^{\beta}$ for all distributive lattices $D$.

This conjecture could be paraphrased as: the class of distributive lattices has fibre size of degree at least $\beta$. More particularly, say that an infinite class $\mathscr{C}$ of ordered sets has fibre degree at least $\beta$ if there is a constant $c>0$ such that, for every $P \in \mathscr{C}$, every fibre in $P$ has size at least $c|P|^{\beta}$. Thus the class of Boolean lattices has fibre degree at least $1 / 3$ and not more than $1 / 2$.

The main barrier to our improving Theorem 1 is our use of the length of a distributive lattice as a lower bound for fibre size, which we consider crude. Thus one might investigate parameters other than length that give better lower bounds on fibre size.

To prove Theorem 1, we play off the dimension of a distributive lattice against its length and invoke a description of partitions induced by maximal antichains in a certain class of distributive lattices [1]. In the process we prove that for at least some distributive lattices, minimal fibre size is a hereditary property: see Theorem 2 . All of this is contained in Section 2, and Theorem 1 itself is proved in Section 3. In Section 4 we give a similar result for powers of chains. Finally, several questions and conjectures are collected in Section 5.

\section{The splitting property}

Our proof of Theorem 1 depends upon showing that minimum fibre size is often a monotone property, where 'monotone' could have more than one meaning, as demonstrated in the following conjecture. In fact, we believe that minimum fibre size is monotone in various respects, within the family of all distributive lattices and perhaps more widely.

CONJECTURE 2. Let $D$ and $M$ be distributive lattices.

(a) If $D$ is a sublattice of $M$ then $f(D) \leq f(M)$.

The example from Section 1 (using $L$ and $L^{\prime}$ ) shows that (a) is not true for arbitrary lattices.

(b) If $D$ is a homomorphic image of $M$ then $f(D) \leq f(M)$.

Might (b) hold for arbitrary lattices? It is not true in general for ordered sets $D$ and $M$ where $D$ is a retract of $M$, since, using the same example, $L$ is a retract of $L^{\prime}$. A special case of both (a) and (b) is

(c) $f(D) \leq f(D \times M)$.

Might (c) hold for arbitrary ordered sets $D$ and $M$ ? 
At this point, we can prove somewhat less than this. Our result, Theorem 2, depends on what Ahlswede, Erdốs and Graham [1] call the 'splitting property' for maximal antichains. Call an ordered set $X$ dense if every nonempty open interval $(a, b)=\{x \in X \mid a<x<b\}$ of $X$ contains at least two elements. Say that a maximal antichain $A$ of $X$ has the splitting property if $A$ can be partitioned into two subsets $B$ and $C$ so that $X=B \uparrow \cup C \downarrow$. [We use $B \uparrow=\{x \in X \mid x \geq b$ for some $b \in B\}$ to denote the order filter generated by $B$ and $C \downarrow=\{x \in X \mid x \leq c$ for some $c \in C\}$ for the order ideal generated by $C$.] $X$ has the splitting property if all of its maximal antichains do. The splitting property for infinite ordered sets is studied in $[7,8]$.

The important results for us from [1] are that Boolean lattices are dense, and every dense ordered set has the splitting property. In fact it is easy to prove that the only dense distributive lattices are Boolean. For suppose $D$ is distributive and the partial order $J(D)$ of join-irreducible elements of $D$ contains two elements $a<b$. Let $A=\{x \in J(D) \mid x<b\} \neq \emptyset$. We can assume that $a$ is a maximal element of $A$. Then in $D$ the open interval $(\bigvee(A-\{a\}), b)$ contains only the element $\bigvee A$, so $D$ is not dense. On the other hand, there are exactly three non-Boolean distributive lattices which have the splitting property. This is the subject of a companion paper [5].

We will now see that the splitting property is nicely tied to the monotonicity of $f$, in all three meanings contained in Conjecture 2. First, we introduce some notation and a lemma. Given an order-preserving map $\Phi$ from an ordered set $P$ onto an ordered set $Q$, and an element $q \in Q$, define

$$
\min \left(q^{-1}\right)=\left\{p \in P: p \text { is a minimal element of } \Phi^{-1}(q)\right\}
$$

and similarly

$$
\max \left(q^{-1}\right)=\left\{p \in P: p \text { is a maximal element of } \Phi^{-1}(q)\right\} .
$$

LEMMA 1. Let $P$ and $Q$ be ordered sets where $Q$ has the splitting property. Suppose there exists an order-preserving map $\Phi$ from $P$ onto $Q$ such that, for all $x \leq y \in Q$,

$$
\text { for all } u \in \max \left(x^{-1}\right) \text { there exists } v \in \max \left(y^{-1}\right) \text { such that } u \leq v
$$

and

$$
\text { for all } v \in \min \left(y^{-1}\right) \text { there exists } u \in \min \left(x^{-1}\right) \text { such that } u \leq v \text {. }
$$

\section{Then}

(a) for any maximal antichain $A$ of $Q$, there is a maximal antichain $A^{\prime}$ of $P$ such that $A=\Phi\left(A^{\prime}\right)\left(=\left\{\Phi(a) \mid a \in A^{\prime}\right\}\right)$;

(b) $f(Q) \leq f(P)$.

ProOF. (a) Let $A$ be a maximal antichain in $Q$. Since $Q$ has the splitting property, 
there are sets $B$ and $C$ which partition $A$ and such that $Q=B \uparrow \cup C \downarrow$. Let

$$
A^{\prime}=\bigcup_{b \in B} \min \left(b^{-1}\right) \cup \bigcup_{c \in C} \max \left(c^{-1}\right) \subseteq P .
$$

Then it is clear that $A^{\prime}$ is an antichain and that $\Phi\left(A^{\prime}\right)=A$. Take any element $x \in P$. Since $A$ has the splitting property in $Q$, we may assume that $\Phi(x)=y \leq c$ in $Q$ for some $c \in C$. Then $x \leq u$ for some $u \in \max \left(y^{-1}\right)$, and by the condition on $\Phi, u \leq v$ for some $v \in \max \left(c^{-1}\right)$. Since $v \in A^{\prime}$ and $x \leq v$, this proves that $A^{\prime}$ is maximal in $P$.

(b) Let $F$ be a fibre in $P$ and let $\widehat{F}=\Phi(F)$. Since $|\widehat{F}| \leq|F|$, it is enough to show that $\widehat{F}$ is a fibre in $Q$. Take $A$ to be a maximal antichain in $Q$ and let $A^{\prime}$ be the corresponding maximal antichain of $P$ as defined in (a). Then, as $F$ is a fibre in $P, F \cap A^{\prime} \neq \emptyset$. We may assume that $u \in F \cap A^{\prime}$ for some $u \in \min \left(b^{-1}\right)$. But then $b=\Phi(u) \in \widehat{F} \cap A$.

Here is what we know so far concerning Conjecture 2.

THEOREM 2. Let $D$ and $M$ be ordered sets, where $D$ has the splitting property.

(a) $f(D) \leq f(D \times M)$.

(b) If $D$ and $M$ are lattices and $D$ is a homomorphic image of $M$, then $f(D) \leq f(M)$.

(c) If $M$ is a distributive lattice and $D$ is an interval sublattice of $M$, then $f(D) \leq$ $f(M)$.

PROOF. (a) As the projection map from $D \times M$ onto $D$ satisfies the properties of $\Phi$ in Lemma 1, this part is immediate.

(b) As any lattice homomorphism of $M$ onto $D$ satisfies the properties of $\Phi$ in Lemma 1 (in fact $\min \left(x^{-1}\right)$ and $\max \left(x^{-1}\right)$ are both singletons for all $x \in D$ ), this part is immediate too.

(c) Assume that $D$ is an interval sublattice of $M$ and that $d_{0}$ is the minimum element of $D$ and $d_{1}$ is the maximum element. Define the map $\Psi$ of $M$ to $D$ by

$$
\Psi(x)=\left(x \vee d_{0}\right) \wedge d_{1}, x \in M .
$$

As $M$ is distributive, $\Psi$ is a homomorphism of $M$ onto $D$. We apply (b) to complete the argument.

It should be pointed out here that, as the lattice $D$ in part (c) is both distributive and has the splitting property, by a separate result [5] $D$ must be either Boolean or isomorphic to $3 \times 2,3 \times 2^{2}$, or $3 \times 2^{3}$. Moreover, if a Boolean lattice $D$ appears as a sublattice of a distributive lattice $M$, then $D$ is an interval sublattice of $M$. The same does not hold for the other distributive lattices with the splitting property, so we must retain 'interval' in (c), though we believe that it is an unnecessary condition in any case. 
We end this section with a result we will not use, but which reveals an interesting connection between the splitting property and maximal antichains.

LEMMA 2. Let $X$ be an ordered set and let $2=\{0,1\}$ with $0<1$. For any maximal antichain $A$ of $X,(A \times\{0\}) \cup(A \times\{1\})$ contains a maximal antichain of $X \times 2$ if and only if $A$ has the splitting property.

Proof. Let $A$ be a maximal antichain of the ordered set $X$. First suppose $A$ has the splitting property. So there are two subsets $B$ and $C$ of $A$ such that $B$ and $C$ partition $A$ and $X=B \uparrow \cup C \downarrow$. Then it is easy to see that $(B \times\{0\}) \cup(C \times\{1\})$ is a maximal antichain in $X \times 2$. Conversely, suppose $(A \times\{0\}) \cup(A \times\{1\})$ contains a maximal antichain of $X \times 2$, which we may write in the form $(B \times\{0\}) \cup(C \times\{1\})$ for subsets $B$ and $C$ of $A$. Clearly $B \cap C=\emptyset$, and since the antichain is maximal it follows easily that $X=B \uparrow \cup C \downarrow$. Thus $A$ has the splitting property.

\section{The proof of Theorem 1}

Let $D$ be an $n$-element distributive lattice of length $l(D)=l$ and dimension $\operatorname{dim}(D)=d$.

Since $D$ has dimension $d$, there is an element of $D$ with precisely $d$ upper covers, and it follows that $D$ has an interval sublattice isomorphic to $2^{d}$. Thus, Theorem 2 (c) and the lower bound on fibre sizes of Boolean lattices obtained in [13] (and to be proven in Section 4) yield

$$
f(D) \geq f\left(2^{d}\right) \geq c(\sqrt[3]{2})^{d}
$$

for some constant $c$.

Again since $D$ has dimension $d, D$ is embeddable as a $0-1$ sublattice in a product of $d$ chains, say of lengths $c_{1}, c_{2}, \ldots, c_{d}$. In particular,

$$
l=c_{1}+c_{2}+\cdots+c_{d} \text { and } n \leq\left(c_{1}+1\right)\left(c_{2}+1\right) \cdots\left(c_{d}+1\right) .
$$

Thus by the arithmetic mean - geometric mean inequality,

$$
\frac{l+d}{d}=\frac{1}{d} \sum_{i=1}^{d}\left(c_{i}+1\right) \geq\left(\prod_{i=1}^{d}\left(c_{i}+1\right)\right)^{1 / d} \geq n^{1 / d}
$$

and so $l \geq d\left(n^{1 / d}-1\right)$. Since $f(D) \geq l(D)$, we have

$$
f(D) \geq c \cdot \max \left((\sqrt[3]{2})^{d}, d\left(n^{1 / d}-1\right)\right) .
$$


To obtain the desired lower bound for $f(D)$ we need to minimize the right hand side of (1) for $d$ between 1 and $\log _{2}(n)$. As a function of $d,(\sqrt[3]{2})^{d}$ is increasing throughout the entire range of values of $d$, namely $1 \leq d \leq \log _{2} n$. Moreover it is easily seen that, treating $d$ as a continuous variable on that domain, $d n^{1 / d}$ (for arithmetic simplicity) takes a unique minimum at $d=\log n$ (the natural $\operatorname{logarithm}$ ). Thus, $\max \left((\sqrt[3]{2})^{d}, d n^{1 / d}\right)$ is minimized for that value of $d$ for which $(\sqrt[3]{2})^{d}=d n^{1 / d}$. We may take $d=\sqrt{3 \log _{2} n}$. Indeed, for $d \geq \sqrt{3 \log _{2} n}$,

$$
(\sqrt[3]{2})^{d} \geq 2^{\frac{1}{3} \sqrt{3 \log _{2} n}}=n^{1 / \sqrt{3 \log _{2} n}} .
$$

Also, note that

$$
d\left(n^{1 / d}-1\right) \geq n^{1 / d} \Longleftrightarrow n \geq\left(\frac{d}{d-1}\right)^{d}=\frac{1}{(1-1 / d)^{d}}
$$

which decreases (to $e$ ) for $d \geq 2$. Thus for $n \geq 4$ and for $2 \leq d \leq \sqrt{3 \log _{2} n}$,

$$
d\left(n^{1 / d}-1\right) \geq n^{1 / d} \geq n^{1 / \sqrt{3 \log _{2} n}} .
$$

Finally, when $d=1$, we have

$$
d\left(n^{1 / d}-1\right)=n-1 \geq n^{1 / \sqrt{3 \log _{2} n}} .
$$

for sufficiently large $n$. By (1) this yields Theorem 1 , since the constant $c$ in the statement can be decreased if necessary to handle all small values of $n$.

\section{Fibres in powers of chains}

We can improve the bound in Theorem 1 (verifying Conjecture 1) for a wider class of distributive lattices than Boolean lattices. Use $\mathbf{m}$ to denote the $m$-element chain ( $m \geq 2$ ), so $\mathbf{m}^{k}$ is the direct product of $k$ copies of $\mathbf{m}$, a $k$-dimensional distributive lattice of cardinality $m^{k}$. Since $2^{k}$ is an interval sublattice of $\mathbf{m}^{k}$, Theorem 2 (c) and the lower bound for $f\left(2^{k}\right)$ due to Euczak noted in Section 1 show that $f\left(\mathbf{m}^{k}\right)$ is of order at least $(\sqrt[3]{2})^{k}$. Using the observations on interval sublattices that support Theorem 2 and types of antichains drawn from [6], we can do better.

For the sake of completeness, we collect all results for powers of chains in the theorem below, including the result for $2^{k}$ due to Luczak. Throughout the remainder of this section we shall set $\mathbf{m}=\{0,1, \ldots, m-1\}$ and use vector notation $\bar{v}=$ $\left(v_{1}, v_{2}, \ldots, v_{k}\right)$, with $v_{i} \in \mathbf{m}$ for members of $\mathbf{m}^{k}$.

THEOREM 3. There is a constant $c>0$ so that for positive integers $k$,

(a) (Luczak) $f\left(2^{k}\right) \geq c\left(2^{k}\right)^{1 / 3}$, 
(b) $f\left(3^{k}\right) \geq c\left(3^{k}\right)^{\log _{3}(3 / 2)} \approx c\left(3^{k}\right)^{3690}$,

(c) $f\left(4^{k}\right) \geq c\left(4^{k}\right)^{\log _{4}(7 / 4)} \approx c\left(4^{k}\right)^{4036}$,

(d) for all $m \geq 5, f\left(\mathbf{m}^{k}\right) \geq c\left(m^{k}\right)^{\log _{m} \sqrt{m-1}}$.

PROOF. The proof of (a) depends on this result from [6]: every fibre in $2^{k}$ contains at least $k ! / 2^{k-1}$ distinct maximal chains. Let $F$ be a fibre of $2^{k}$ and suppose that $|F|=\alpha^{k}$. We show that $\alpha \geq \sqrt[3]{2}$. It makes the arithmetic simpler to assume that $k$ is divisible by 4 .

Since each of the $k$ ! maximal chains of $2^{k}$ contains an element with $k / 2$ l's and $F$ has at most $\alpha^{k}$ such elements, there must be some $\bar{a} \in F$ with $k / 21$ 's and so that the family $\mathscr{M}$ of all maximal chains contained in $F$ and containing $\bar{a}$ satisfies

$$
|\mathscr{M}| \geq \frac{k ! / 2^{k-1}}{\alpha^{k}} .
$$

Let $\mathscr{M}_{0}\left(\mathscr{M}_{1}\right)$ denote the set of all restrictions of chains in $\mathscr{M}$ to the interval $[\overline{0}, \bar{a}]$ (respectively, $[\bar{a}, \overline{1}]$ ). Without loss of generality, from (2) we may deduce that

$$
\left|\mathscr{M}_{0}\right| \geq\left(k ! /(2 \alpha)^{k}\right)^{1 / 2}
$$

Since $F$ contains at most $\alpha^{k}$ elements with $k / 41$ 's, each member of $\mathscr{M}_{0}$ contains one of these elements, and each such element belongs to $((k / 4) !)^{2}$ maximal chains in the interval $[\overline{0}, \bar{a}]$,

$$
\alpha^{k}((k / 4) !)^{2} \geq\left|\mathscr{M}_{0}\right| \geq\left(k ! /(2 \alpha)^{k}\right)^{1 / 2} .
$$

It follows easily from (4) that

$$
\alpha^{3 k} \geq \frac{k !}{2^{k}((k / 4) !)^{4}} .
$$

Now if we use Stirling's approximation $n ! \approx(n / e)^{n}$, the right-hand side of (5) becomes

$$
\frac{(k / e)^{k}}{2^{k}(k /(4 e))^{k}}=2^{k}
$$

and from (5) we get $\alpha \geq \sqrt[3]{2}$ as claimed. More precisely, we know that for any positive $a>e>b$,

$$
(n / a)^{n}<n !<(n / b)^{n}
$$

for all sufficiently large $n$. Thus by (5), for any fixed positive $a>e>b$,

$$
\alpha^{3 k} \geq \frac{(k / a)^{k}}{2^{k}(k /(4 b))^{k}}=\left(\frac{2 b}{a}\right)^{k}
$$


for sufficiently large $k$. Thus $\alpha^{3} \geq 2 b / a$. Since this is true for all $a>e>b$, we must have $\alpha^{3} \geq 2$, and thus $\alpha \geq \sqrt[3]{2}$.

Next, the proofs of (b) and (c) can be obtained with the one approach. For each $\bar{v} \in \mathbf{m}^{k}$ with $\bar{v}=\left(v_{1}, v_{2}, \ldots, v_{k}\right)$ satisfying $v_{i} \leq m-2$ for all $i$, let $\overline{v+1}=$ $\left(v_{1}+1, v_{2}+1, \ldots, v_{k}+1\right)$. Then the interval $[\bar{v}, \overline{v+1}] \cong \mathbf{2}^{k}$. For each such $\bar{v}$ and for $l$ with $1 \leq l<k / 2$, we consider all pairs $(\bar{v}, \bar{a})$ where $\bar{a}$ has height $2 l-1$ in the interval $[\bar{v}, \overline{v+1}]$. Obviously, there are $(m-1)^{k}\left(\begin{array}{c}k \\ 2 l-1\end{array}\right)$ such pairs.

For each pair $(\bar{v}, \bar{a})$, we obtain an antichain $A(\bar{v}, \bar{a})$ maximal within $[\bar{v}, \overline{v+1}]$ as in [6, Theorem 2]: $A(\bar{v}, \bar{a})=B(\bar{v}, \bar{a}) \cup C(\bar{v}, \bar{a})$ where

$$
B(\bar{v}, \bar{a})=\{\bar{w} \mid \bar{v}<\bar{w}<\bar{a}, \bar{w} \text { has height } l \text { in }[\bar{v}, \overline{v+1}]\},
$$

and

$$
C(\bar{v}, \bar{a})=\left\{\bar{w}^{\prime} \mid \bar{w}^{\prime} \text { is the complement in }[\bar{v}, \overline{v+1}] \text { of some } \bar{w} \in B(\bar{v}, \bar{a})\right\} .
$$

To show that $A(\bar{v}, \bar{a})$ is an antichain, suppose $\bar{w} \in B(\bar{v}, \bar{a})$ and $\bar{z}^{\prime} \in C(\bar{v}, \bar{a})$ where $\bar{z} \in B(\bar{v}, \bar{a})$. Then $w_{i}=a_{i}=v_{i}+1$ for $l$ coordinates $i$ and $z_{j}=a_{j}=v_{j}+1$ for $l$ coordinates $j$, while $\bar{w} \leq \bar{z}^{\prime}$ would say that the $i$ 's and $j$ 's must be distinct, which means that $\bar{a}$ agrees with $\overline{v+1}$ in at least $2 l$ coordinates, a contradiction since the height of $\bar{a}$ in $[\bar{v}, \overline{v+1}]$ is only $2 l-1$. To show that $A(\bar{v}, \bar{a})$ is maximal, for any $\bar{y} \in[\bar{v}, \overline{v+1}]$, either $y_{i}=a_{i}=v_{i}+1$ for at least $l$ coordinates $i$, in which case $\bar{w} \leq \bar{y}$ for some $\bar{w} \in B(\bar{v}, \bar{a})$; or else $y_{i}=v_{i}$ and $a_{i}=v_{i}+1$ for some $l$ coordinates $i$, in which case $\bar{y} \leq \bar{z}^{\prime}$ for some $\bar{z} \in B(\bar{v}, \bar{a})$, namely where $z_{j}=v_{j}+1$ exactly on these $l$ coordinates.

This argument shows that, within the interval $[\bar{v}, \overline{v+1}]$, the maximal antichain $A(\bar{v}, \bar{a})$ has the splitting property: $[\bar{v}, \overline{v+1}] \subseteq B(\bar{v}, \bar{a}) \uparrow \cup C(\bar{v}, \bar{a}) \downarrow$. So, just as in the proof of Lemma 1 (a), the homomorphism $\Psi_{\bar{v}}$ of $\mathbf{m}^{k}$ to $[\bar{v}, \overline{v+1}]$ defined by

$$
\Psi_{\bar{v}}(\bar{x})=(\bar{x} \vee \bar{v}) \wedge(\overline{v+1}), \quad \bar{x} \in \mathbf{m}^{k},
$$

yields a maximal antichain $A^{\prime}(\bar{v}, \bar{a})$ of $\mathbf{m}^{k}$.

Given any $\bar{u} \in \mathbf{m}^{k}$, we determine the number of maximal antichains of the form $A^{\prime}(\bar{v}, \bar{a})$ to which $\bar{u}$ can belong. Suppose that $\bar{u} \in A^{\prime}(\bar{v}, \bar{a})$ and that $\Psi_{\bar{v}}(\bar{u})=\bar{w} \in$ $B(\bar{v}, \bar{a})$, that is, where $\bar{w}$ has height $l$ in $[\bar{v}, \overline{v+1}]$. Recall that this means that $\bar{u}$ is the minimum element of its $\Psi_{\tilde{v}}$-congruence class. Then we claim

(i) $w_{i}=v_{i}+1$ implies that $w_{i}=u_{i}$, and

(ii) $w_{i}=v_{i}$ implies that $\boldsymbol{u}_{i}=0$.

For (i), if $u_{i}>w_{i}$, define $\overline{\boldsymbol{u}}^{\prime}$ by $\boldsymbol{u}_{i}^{\prime}=w_{i}$ and $u_{j}^{\prime}=u_{j}$ for $j \neq i$; then $\overline{\boldsymbol{u}}^{\prime}<\overline{\boldsymbol{u}}$, but one calculates that $\left(\bar{u}^{\prime} \vee \bar{v}\right) \wedge(\overline{v+1})=\bar{w}$, contradicting that $\bar{u}$ is minimal in $\Psi_{\bar{v}}^{-1}(\bar{w})$. If $u_{i}<w_{i}=v_{i}+1$, then $\left(u_{i} \vee v_{i}\right) \wedge\left(v_{i}+1\right)=v_{i} \neq w_{i}$, contradicting $\Psi_{\bar{v}}(\bar{u})=\bar{w}$. Thus $u_{i}$ must equal $w_{i}$. 
For (ii), if $u_{i}>0$ then define $\bar{u}^{\prime}$ by $u_{i}^{\prime}=0$ and $u_{j}^{\prime}=u_{j}$ for $j \neq i$; then, as in (i), $\bar{u}^{\prime}<\bar{u}$ and $\left(\bar{u}^{\prime} \vee \bar{v}\right) \wedge(\overline{v+1})=\bar{w}$, contradiction. So $u_{i}=0$. This finishes the proof of the claim.

Moreover, (i) holds for $l$ indices $i$, and (ii) holds for $k-l$ indices $i$. So, with $\bar{u}$ given, for each $i$ such that $u_{i} \neq 0, u_{i}=w_{i}=v_{i}+1$, that is, $v_{i}$ is determined. For all other indices $i, v_{i}$ can take any value in $\{0,1, \ldots, m-2\}$. Thus, there are $(m-1)^{k-1}$ choices for $\bar{v}$.

Given $\bar{v}$ and thus $\bar{w}=(\bar{u} \vee \bar{v}) \wedge(\overline{v+1})$, there are $\left(\begin{array}{c}k-1 \\ l-1\end{array}\right)$ choices of $\bar{a}$ such that $\bar{u} \in A^{\prime}(\bar{v}, \bar{a})$. Thus each $\bar{u} \in \mathbf{m}^{k}$ can belong to at most $(m-1)^{k-l}\left(\begin{array}{c}k-l \\ l-1\end{array}\right)$ maximal antichains of the form $A^{\prime}(\bar{v}, \bar{a})$. We have actually argued that each $\bar{u}$ can be the minimum element of $\Psi_{\bar{v}}^{-1}(\bar{w})$, with $\bar{w} \in B(\bar{v}, \bar{a})$ for at most this number of pairs $(\bar{v}, \bar{a})$. Moreover, it is not possible for $\bar{u}$ to be minimum in some $\Psi_{\bar{v}}^{-1}(\bar{w})$ and maximum in some $\Psi_{\bar{v}^{\prime}}^{-1}\left(\bar{w}^{\prime}\right)$ since $\bar{u}$ would have $k-l$ entries 0 and $k-l$ entries $m-1$, meaning that $2 k-2 l \leq k$ and so $k \leq 2 l$, contradicting $l<k / 2$.

We have shown that every fibre of $\mathbf{m}^{k}$ must have size at least

$$
\frac{(m-1)^{k}\left(\begin{array}{c}
k \\
2 l-1
\end{array}\right)}{(m-1)^{k-l}\left(\begin{array}{c}
k-l \\
l-1
\end{array}\right)}=\frac{\left(\begin{array}{l}
k \\
l
\end{array}\right)}{\left(\begin{array}{c}
2 l-1 \\
l
\end{array}\right)}(m-1)^{l}=g(l) .
$$

With the function $g(l)$ as defined in (6), an easy calculation yields

$$
\frac{g(l+1)}{g(l)}=\frac{(k-l)(m-1)}{4 l+2} .
$$

We can use (7) to determine, for given $m$, which value of $l$ maximizes $g(l)$. The right side of (7) exceeds 1 if and only if

$$
l \leq \frac{k(m-1)-2}{m+3},
$$

so $g(l)$ will be maximized when

$$
l=\frac{k(m-1)-2}{m+3} .
$$

Setting $l=\lambda k$ and applying Stirling's formula again, we obtain this expression for $g(l)$ from (6):

$$
g(\lambda k)=2\left(\frac{(m-1)^{\lambda}}{(4 \lambda)^{\lambda}(1-\lambda)^{1-\lambda}}\right)^{k} .
$$

From (8), we see we want

$$
\lambda=(m-1) /(m+3),
$$

if this lies in the acceptable range for $\lambda$. 
Thus, to obtain (b), set $m=3$ and get $\lambda=1 / 3$. Inserting these values in (9), we obtain

$$
f\left(3^{k}\right) \geq g(k / 3)=c(3 / 2)^{k}=c\left(3^{k}\right)^{\log _{3}(3 / 2)} .
$$

To obtain (c), set $m=4$ to get $\lambda=3 / 7$ which when inserted in (9) yields

$$
f\left(4^{k}\right) \geq g(3 k / 7)=c(7 / 4)^{k}=c\left(4^{k}\right)^{\log _{4}(7 / 4)} .
$$

To obtain (d), we can use a simpler argument. Instead of using antichains of the form $A(\bar{v}, \bar{a})=B(\bar{v}, \bar{a}) \cup C(\bar{v}, \bar{a})$ in the interval $[\bar{v}, \overline{v+1}]$, for each $\bar{v}=\left(v_{1}, v_{2}, \ldots, v_{k}\right)$ with $v_{i} \leq m-2$ for all $i$, take $A(\bar{v})$ to be the 'middle level' of $[\bar{v}, \overline{v+1}]$, that is, the maximal antichain of all elements in $[\bar{v}, \overline{v+1}]$ with $k / 21$ 's. Define $\Psi_{\bar{v}}$ as above to obtain a maximal antichain $A^{\prime}(\bar{v})$ of $\mathbf{m}^{k}$. Then suppose $\bar{u} \in \mathbf{m}^{k}$ is the minimum element of $\Psi_{\bar{v}}^{-1}(\bar{w})$ for some $\bar{v} \in \mathbf{m}^{k}$ and some $\bar{w} \in A(\bar{v})$. It is easy to check that, as before, if $u_{i}=0$ then $v_{i}$ is arbitrary and $w_{i}=v_{i}$ (which occurs for $k / 2$ indices $i$ ), while if $u_{i}>0$ then we must have $w_{i}=v_{i}+1=u_{i}$. Thus, any $\bar{u}$ in $\mathbf{m}^{k}$ can be the minimum element in some $\Psi_{\bar{v}}^{-1}(\bar{w}), \bar{w} \in A(\bar{v})$, for at most $(m-1)^{k / 2}$ elements $\bar{v}$, and the maximal element for at most an equal number of $\bar{v}$ 's, and so can occur in at most $2(m-1)^{k / 2} A^{\prime}(\bar{v})$ 's. Since there are $(m-1)^{k} A^{\prime}(\bar{v})$ 's, this shows that any fibre in $\mathbf{m}^{k}$ has size at least

$$
\frac{1}{2}(m-1)^{k / 2}=\frac{1}{2}\left(m^{k}\right)^{\log _{m} \sqrt{m-1}},
$$

which completes the proof.

(The argument in (d) also applies to the cases $m=4$ and 3, but results in weaker bounds than we obtained doing it the harder way.)

COROLLARY 1. The class of all powers $\mathbf{m}^{k}$ of chains, where $m \geq 2$ and $k \geq 1$, has fibre degree at least $1 / 3$.

ProOF. The result follows from the above theorem and from the facts that $\log _{5}(2)>$ $1 / 3$ and the function $\log _{m} \sqrt{m-1}$ is increasing for $m>1$.

It is interesting to see how the lower bounds in Theorem 3 compare to the sizes of fibres we can find in $\mathbf{m}^{k}$. We again rely on fibres of the form $C(\bar{v}), \bar{v} \in \mathbf{m}^{k}$. Since

$$
|C(\bar{v})|=\left[\prod_{i=1}^{k}\left(v_{i}+1\right)\right]+\left[\prod_{i=1}^{k}\left(m-v_{i}\right)\right]-1,
$$

choosing $k / 2$ entries $v_{i}=0$ and $k / 2$ entries $v_{i}=m-1$ gives $|C(\bar{v})|=2 m^{k / 2}-1$. Thus, since $\lim _{m \rightarrow \infty} \log _{m} \sqrt{m-1}=1 / 2$, Theorem 3 (d) just about determines the order of the minimum fibre size of $\mathbf{m}^{k}$ for large $m$. 


\section{Further problems}

We believe that Boolean lattices should have, at least for large enough $n$, the smallest minimum-sized fibres among distributive lattices of size $n$.

CONJECTURE 3. Among all distributive lattices on $n=2^{k}$ elements, the Boolean lattice has the minimum fibre size.

This belief stems from a vague 'principle' that Boolean lattices are the 'shortest' and 'widest' of distributive lattices, and that as a consequence certain results true for Boolean lattices should hold for all distributive lattices. In these instances Boolean lattices should afford the 'extremal' examples of these results. For an earlier example where this principle is illustrated, see [9] and the subsequent paper [11]. In [9] it is conjectured that $|L| \geq O(w \sqrt{\log w})$ for all distributive lattices $L$ of width $w$. Note that Boolean lattices give equality in this inequality. This conjecture remains unsettled, though a weaker bound is proved in [11].

As was mentioned in Section 1, for Boolean lattices the smallest known fibres are of the type $C(x)$, the family of all sets comparable to $x$, where $x$ is a 'middle-sized' subset. This gives a fibre of size essentially the square root of the size of the Boolean lattice [12]. We now show that Conjecture 3 will not be disproved by looking at fibres $C(x)$ of any distributive lattice.

Lemma 3. Let $D$ be a distributive lattice with $n$ elements and let $x \in D$. Then $|C(x)| \geq 2 \sqrt{n}-1$.

ProOF. Let $a=|x \uparrow|$ and let $b=|x \downarrow|$. Then $|C(x)|=a+b-1$. The mapping $\phi$ of $D$ to $x \uparrow \times x \downarrow$ defined by

$$
\phi(z)=(x \vee z, x \wedge z)
$$

is a lattice embedding of $D$ in the direct product $x \uparrow \times x \downarrow$. This follows from the distributivity of $D$. So

$$
n=|D| \leq|x \uparrow \times x \downarrow|=a b,
$$

and thus by the arithmetic mean-geometric mean inequality,

$$
|C(x)|=a+b-1 \geq 2 \sqrt{a b}-1 \geq 2 \sqrt{n}-1 .
$$

Observe that for one class of distributive lattices, the minimum fibre size is easily determined, and equals $|C(x)|$ for some element $x$. Take $D=A \times B$ where $A$ is an $a$-element chain and $B$ is a $b$-element chain. Then $C\left(\left(1_{A}, 0_{B}\right)\right)$ has $a+b-1$ elements, and every fibre must have at least this many elements as this is the size of any maximal 
chain of $D$. So by Lemma $3, f(D)=a+b-1 \geq 2 \sqrt{|D|}-1$. In fact for $a=b$ we have equality.

To do better than $\sqrt{n}$ as an upper bound for $f(D)$ for some $n$-element distributive lattice $D$, we would need to obtain other classes of (minimal) fibres.

PROBLEM 1. For families of ordered sets (distributive lattices, Boolean lattices), find examples of (minimal) fibres other than the sets of all elements comparable to a fixed element.

Zita Cheng, until recently an undergraduate student at the University of Calgary, has shown in unpublished work that the minimum-sized fibre for lattices of the form $\mathbf{2} \times \mathbf{b} \times \mathbf{c}$ is such a set $C(x)$, but that this is not true for $3^{3}$; the smallest subset $C(x)$ of this lattice has 11 elements, while there is a fibre of only 10 elements.

Finally, one way to ensure that every fibre of an ordered set $P$ has at least $N$ elements is to find $N$ pairwise disjoint maximal antichains of $P$. It was proved in [6] that, for every sufficiently large $n$, the Boolean lattice $L=2^{n}$ contains $1.0674422^{n}=$ $|L|^{0.094157952}$ pairwise disjoint maximal antichains.

CONJECTURE 4. There is a constant $c>0$ so that every distributive lattice $D$ contains $|D|^{c}$ pairwise disjoint maximal antichains.

This is, of course, not true for all lattices. The modular nondistributive lattice $M_{n}$ (length 2 , with $n$ atoms) contains only 3 maximal antichains altogether.

\section{References}

[1] R. Ahlswede, P. L. Erdốs, and N. Graham, 'A splitting property of maximal antichains', Combinatorica 15 (1995), 475-480.

[2] M. Aigner and T. Andreae, 'Vertex sets that meet all maximal cliques of a graph', preprint (1986).

[3] B. Bajnok, 'On the minimum width of a cutset in the truncated Boolean lattice', Congr. Numer. 130 (1998), 77-81.

[4] B. Bajnok and S. Shahriari, 'Long symmetric chains in the Boolean lattice', J. Combin. Theory Ser. A 75 (1996), 44-54.

[5] D. Duffus and B. Sands, 'Finite distributive lattices with the splitting property', in preparation.

[6] D. Duffus, B. Sands, and P. Winkler, 'Maximal chains and antichains in Boolean lattices', SIAM J. Disc. Math. 3 (1990), 197-205.

[7] M. Džamonja, 'A note on the splitting property in strongly dense posets of size $\aleph_{0}$ ', Radovi Matematički 8 (1999), 321-326.

[8] P. L. Erdốs, 'Splitting property in infinite posets', Discrete Math. 163 (1997), 251-256.

[9] U. Faigle and B. Sands, 'A size-width inequality for distributive lattices', Combinatorica 6 (1986), 29-33.

[10] Z. Füredi, J. Griggs, and D. Kleitman, 'A minimal cutset of the Boolean lattice with almost all members', Graphs Combin. 5 (1989), 327-333. 
[11] J. Kahn and M. Saks, 'On the widths of finite distributive lattices', Discrete Math. 63 (1987), 183-195.

[12] Z. Lonc and I. Rival, 'Chains, antichains and fibres', J. Combin. Theory Ser. A 44 (1987), 207-228.

[13] T. Luczak, private communication, March 1998.

Mathematics and

Computer Science Department

Emory University

Atlanta GA 30322

USA

e-mail: dwight@mathcs.emory.edu
Mathematics and Statistics Department The University of Calgary Calgary AB T2N 1N4

Canada

e-mail: sands@math.ucalgary.ca 